Thomas A. Schmitz*

\title{
Warum gibt es Literatur? Griechische Bibliotheken, römische Leser und die Entwicklung einer literarischen Kultur
}

https://doi.org/10.1515/bfp-2021-0019

Zusammenfassung: Das Aufkommen einer Buchkultur und die Gründung großer Bibliotheken waren seit dem 3. Jh. v. Chr. entscheidend für die Etablierung einer literarischen Tradition. Ihr begegnet die römische Kultur seit dem 2. Jh. v. Chr. in Gestalt von Büchern und Bibliotheken. Dies hat nicht nur in Rom Vorstellungen von der Arbeitsweise eines Autors und den Lektüreerfahrungen von Lesern entscheidend geprägt, sondern bleibt wichtig für das Verständnis dafür, was Literatur ist.

Schlüsselwörter: Griechenland; Rom; Hellenismus; Lesekultur; Tradition; Autor

\section{Why Does Literature Exist? Greek Libraries, Roman Rea- ders, and the Emergence of Literate Culture in Rome}

Abstract: The emergence of a literate culture and the foundation of libraries since the 3rd century BC played a prominent part in establishing a literary tradition. This tradition entered Roman culture in the form of books and libraries. This had repercussions for Roman ideas about authors and readers; they remain important for our own expectations about literature.

Keywords: Greece; Rome; Hellenistic; literate culture; tradition; author

Im Jahr 168 v. Chr. besiegte der römische Feldherr Lucius Aemilius Paullus bei Pydna den makedonischen König Perseus. Der Sieg war kriegsentscheidend, fügte den Makedonen hohe Verluste zu und beendete die makedonische Vorherrschaft in Griechenland. Nach der Schlacht, die im Juni des Jahres stattgefunden hatte, blieb Aemilius Paullus noch eine Weile in Griechenland, wobei er elegant Plünderungen seiner Truppen in großem Maßstab mit einer Art Kulturtourismus verband, der ihn an wichtige Stätten Griechenlands wie Delphi und Olympia führte. Eines unserer Hauptzeugnisse für diese Ereignisse ist die von Plutarch

*Kontaktperson: Prof. Dr. Thomas A. Schmitz, thomas.schmitz@uni-bonn.de (ca. 45-120) geschriebene Biografie des Aemilius Paullus, in der dieser als Muster von Gerechtigkeit und Selbstbeherrschung dargestellt wird. Diese Eigenschaften zeigt er auch nach seinem Sieg (Plutarch, Leben des Aemius Paullus 28, 10-11):

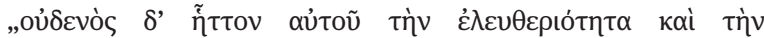

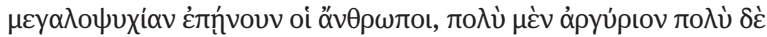

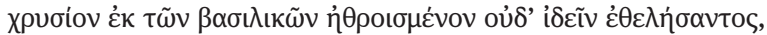

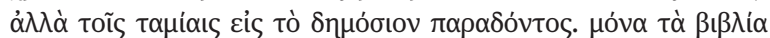

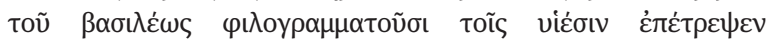

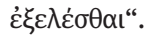

„Insbesondere lobten die Menschen seine Großzügigkeit und Aufrichtigkeit, denn als aus den Schätzen des Königs viel Silber und Gold eingesammelt worden war, wollte er es nicht einmal anschauen, sondern übergab es den Quaestoren für die Staatskasse. Einzig die Bücher des Königs erlaubte er seinen Söhnen anzunehmen, denn sie waren Literaturliebhaber“.

Obwohl der Sieg über Perseus ihm und seinen Truppen überreiche Beute präsentiert, möchte er selbst für sich nichts annehmen. Eine einzige Ausnahme macht er: Die Bibliothek des Königs nimmt er, gewissermaßen als Mitbringsel von einer langen Dienstreise, seinen Söhnen mit. Beide konnten übrigens von diesem reichen Geschenk nicht mehr profitieren; sie starben wenige Tage vor bzw. nach dem Triumphzug ihres Vaters in Rom, was Aemilius Paullus für Plutarch zu einem Musterbeispiel dafür macht, dass im menschlichen Leben Glück und Unglück nicht in reiner Form vorkommen.

Die kleine Anekdote aus Plutarch eignet sich gut dazu, einen Einstieg in die große Frage „Warum gibt es Literatur?“ zu finden. Die Ursprünge unserer abendländischen Vorstellung von Literatur sind in der griechisch-römischen Antike $\mathrm{zu}$ finden, und Bibliotheken haben bei ihrer Entwicklung eine wichtige Rolle gespielt: Ohne große Büchersammlungen wären unsere Auffassungen davon, was eine Autorin oder ein Autor ist und wie sie arbeiten, ganz andere. Um dies besser zu verstehen, soll im Folgenden knapp die Vorgeschichte von Plutarchs Anekdote skizziert werden (warum besaß König Perseus überhaupt eine große Bibliothek?) sowie ihre Konsequenzen für das literarische Leben in Rom (was fingen Römer mit einer griechischen Bibliothek an?). Daran werden sich Gedanken darüber 
anschließen, welchen Beitrag solche Bibliotheken zu neuzeitlichen Vorstellungen von Literatur geleistet haben.

Was also bewog einen makedonischen König, eine Bibliothek anzulegen? Dichtung war in der Frühzeit Griechenlands, also etwa zwischen dem 8. und dem 6. Jh. v. Chr., vorwiegend in einer Performance- und Gesangskultur produziert und rezipiert worden. ${ }^{1}$ Dichterische Texte wurden $\mathrm{zu}$ bestimmten Anlässen in einer Gemeinschaft von Zuhörern aufgeführt und wahrgenommen; Beispiele für solche Gelegenheiten sind etwa religiöse Feiern, städtische Feste, aber auch private Anlässe wie Symposien. Vorgetragen wurden die Texte häufig von den Dichtern selbst; wenn ein Chor die Gedichte vortrug, so studierte oftmals der Dichter selbst ihn ein. Die Alphabetschrift war spätestens im 8. Jh. v. Chr. in Griechenland eingeführt worden; ${ }^{2}$ dass wir noch Texte aus dieser archaischen Kultur überliefert haben, ist ein klares Indiz dafür, dass die Dichter selbst sich bereits der Schrift bedienten, um ihre Texte zu komponieren und $\mathrm{zu}$ archivieren. Aber diese Schriftfassungen dienten lediglich als Grundlage für die mündliche Aufführung, und für das Publikum war diese Performance das Gedicht, nicht ein schriftlicher Text (ähnlich wie heute für das Publikum eine Beethoven-Sinfonie ihre musikalische Realisierung im Konzert ist, nicht das Notenbild der Partitur).

Dieser Zustand ändert sich im Lauf des 5. Jh. v. Chr.: Jetzt wird Lesen und Schreiben immer mehr zum Bestandteil des alltäglichen Lebens. ${ }^{3}$ Zahlreiche Vasenbilder zeigen uns Lesende und Schulszenen, in denen Kinder Lesen und Schreiben lernen. ${ }^{4}$ Der Historiker Herodot berichtet $(6,27,1)$, das kurz vor dem Ionischen Aufstand des Jahres 495 v. Chr. auf der Insel Chios eine Schule einstürzte und 120 Kinder erschlug. Chios war in dieser Zeit reich; dennoch ist die Anzahl von Schülern bemerkenswert: Schulunterricht war nichts Außergewöhnliches mehr, sondern die Regel. So etwas wie eine Schulpflicht oder ein öffentlich finanziertes Bildungssystem gab es in Griechenland nicht; dennoch scheint ein erheblicher Teil der Bürger ihre Kinder zur Schule geschickt zu haben, damit sie dort Lesen und Schreiben lernten.

Diese zunehmende Alphabetisierung beschleunigte sich insbesondere in Athen: Die Stadt war nach dem Sieg der Griechen gegen die Perser 480/479 v.Chr. zum kulturellen und wirtschaftlichen Zentrum Griechenlands geworden und zog viele Intellektuelle an. Hier hören wir in den letzten Jahrzehnten des 5. Jh. von der Existenz eines

1 Dazu bleibt lesenswert Herington (1985) 3-76.

2 Thomas (1999), Powell (2009).

3 Vgl. etwa Pébarthe (2006).

4 Immerwahr (1964), Immerwahr (1973).
Buchhandels, hier begegnen wir zum ersten Mal Menschen, die Literatur lesend aufnahmen und literarische Texte zu sammeln begannen. Aus der Fülle von Zeugnissen seien lediglich wenige Beispiele zitiert. Xenophon berichtet in seinen zu Beginn des 4. Jh. v.Chr. entstandenen Erinnerungen an Sokrates von dem jungen Euthydem, der so viele Bücher sammelt, wie er nur finden kann (4, 2, 1-8):

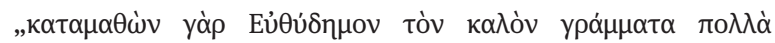

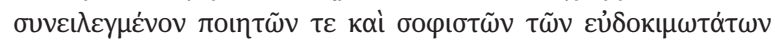

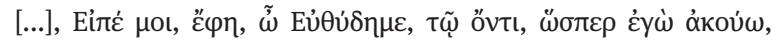

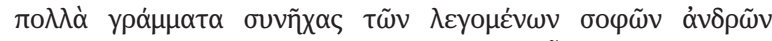

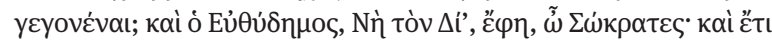

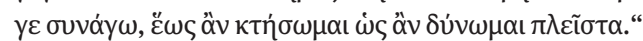

„[Sokrates] erfuhr, der schöne Euthydem habe viele Schriften der berühmtesten Dichter und Sophisten gesammelt. [...] Er fragte ihn: ,Euthydem, sage mir, hast du wirklich, wie ich erfahren habe, viele Schriften der Männer gesammelt, die weise genannt werden?‘ Euthydem antwortete: ,Ja bei Zeus, und ich sammle immer noch, bis ich so viele wie möglich besitze!““

Hier scheint also eine Büchersammlung zu entstehen, eine private Bibliothek, allerdings zu einer Zeit, als so etwas noch außergewöhnlich ist und zu Nachfragen Anlass gibt. Gegen dieselbe Zeit hören wir auch von der Existenz eines Buchhandels in Athen: Der komische Dichter Eupolis, der gegen 410 starb, erwähnt einen Abschnitt des Marktplatzes, auf dem „Bücher käuflich sind“ (frg. 327 Poetae Comici Graeci); dort konnte man für wenig Geld u.a. die philosophische Abhandlung des Anaxagoras erwerben, wie Platon in seiner Apologie des Sokrates ( $26 \mathrm{~d}$-e) berichtet, deren dramatisches Datum der Prozess des Sokrates 399 v. Chr. ist. Wiederum bei Xenophon, in seiner Anabasis $(7,5,14)$, erfahren wir, dass es am Ende des 5. Jh. bereits einen „internationalen“ Buchhandel gab: Bei ihrem Marsch von Persien zum Schwarzen Meer finden die griechischen Söldner um Xenophon am Strand von Salmydessos unter Schiffstrümmern auch „beschriebene Bücher“, die offenbar aus Athen in die griechischen Städte im Schwarzmeergebiet exportiert wurden.

In welchem Maß wir auch schon für das 5. Jh. mit der Existenz großer Bibliotheken rechnen dürfen, ist in der Forschung eine offene Frage. Wir besitzen einige späte Nachrichten darüber, dass schon zu Beginn dieses Jahrhunderts der athenische Tyrann Peisistratos sowie im späten 6. Jh. Polykrates, der aus Schillers Ballade bekannte Tyrann der Insel Samos, Bibliotheken besessen haben sollen (Athenaios, Die Deipnosophisten Buch 1, 3 a; der Text stammt aus dem 2. Jh. n. Chr.), aber nicht nur unterliegt die Zuverlässigkeit dieser Überlieferung Zweifeln; angesichts der oben skizzierten Entwicklungen, wonach Alphabetisierung und Buchhandel vor allem Phänomene des ausgehenden 5. Jh. sind, fragt man sich auch, wie groß solche 
Sammlungen überhaupt gewesen sein können. Blanck ${ }^{5}$ vermutet, dass an den Höfen solcher Herrscher anwesende Dichter „Dedikationsexemplare ihrer Werke“ übergeben haben könnten; das würde auf recht kleine Sammlungen von Manuskripten hindeuten, die eher Schatzkammern als Bibliotheken waren. Wichtiger als geschriebene Bücher scheint jedenfalls für die „Kulturpolitik“ solcher Herrscher die Aufführung von Dichtung gewesen zu sein. So haben wir etwa von dem Dichter Ibykos umfangreiche Bruchstücke eines Lieds (S 151 Poetarum Melicorum Graecorum Fragmenta), in dem er den Tyrannen Polykrates preist und mit den Heroen des trojanischen Kriegs vergleicht. Für Herrscher in dieser Zeit (insbesondere für solche, deren Legitimität nicht über jeden Zweifel erhaben war) stellten solche Gedichte eine Form der Propaganda dar; durch Wiederaufführung und Verbreitung in der griechischsprachigen Welt konnten sie den Ruhm des Herrschers verbreiten.

An diese Form des Mäzenatentums und der Kulturpolitik konnten dann rund 200 Jahre später die hellenistischen Herrscher anknüpfen, deren literarisches Engagement in einer politisch, sozial und kulturell grundlegend veränderten Welt stattfand. Der junge makedonische König Alexander hatte nach seinem Machtantritt 336 v. Chr. in wenigen Jahren einen großen Teil der damals bekannten Welt bis an die Grenzen Indiens erobert; bei seinem frühen Tod 323 in Babylon hinterließ er allerdings kein politisch stabiles Reich, sondern die eroberten Gebiete zerfielen bald in eine Anzahl von Diadochenstaaten, die von seinen Heerführern regiert wurden und sich in ständigen Kriegen miteinander und mit ihren Nachbarstaaten aufrieben. Bleibender hingegen war die kulturelle Hinterlassenschaft seiner Feldzüge: In den eroberten Gebieten etablierte sich eine gräzisierende Kultur, die insbesondere auf die sozialen Eliten eine große Anziehungskraft ausübte. Griechische Architektur und griechische Kunst, griechische Wissenschaft und griechische Ingenieurskunst, griechische Philosophie und griechische Literatur waren in weiten Teilen Ägyptens, Nordafrikas, des Vorderen und Mittleren Orients ebenso verbreitet wie in Griechenland selbst und den traditionell griechisch kolonisierten Gegenden Süditaliens und Siziliens.

In dieser kulturellen Umgebung entstehen etwa seit dem Beginn des 3. Jh. v. Chr. in zahlreichen Großstädten auch Bibliotheken, oftmals direkt finanziert und organisiert von den hellenistischen Machthabern. Die zweifelsohne bedeutendste und berühmteste findet sich in Alexandria; ${ }^{6}$ über die teils skrupellosen Mittel, mit denen die

5 Blanck (1992) 134.
Machthaber in Ägypten, die Ptolemäer, diese Sammlung pflegten und erweiterten, kursierten schon in der Antike zahlreiche teils legendäre Anekdoten. ${ }^{7}$ Eine rivalisierende Bibliothek entstand im 2. Jh. v. Chr. in Pergamon; auch in der seleukidischen Hauptstadt Antiocheia gab es eine Bibliothek. ${ }^{8}$

Durch solche Bibliotheksgründungen setzen die hellenistischen Herrscherhäuser das kulturelle Mäzenatentum früherer griechischer Tyrannen fort, und sie tun es mit den Mitteln ihrer Zeit: An die Stelle von aufgeführter Dichtung tritt nun das geschriebene Buch, das in der über einen großen geografischen Raum verstreuten griechischen Kultur leichter verpflanzbar ist als eine Fest- und Gesangskultur. Damit soll nicht gesagt sein, dass die Aufführung von Dichtung im Zeitalter des Hellenismus gänzlich endete und überhaupt keine Rolle mehr spielte. Auch in dieser Epoche wurde Theater gespielt, wurden Chöre aufgeführt, wurden beim Symposion Gedichte zur Lyra vorgetragen. Aber neben dieser Performance existiert nun auch die private Lektüre als Mittel der Rezeption von Literatur, unabhängig von Zeit und Ort öffentlicher oder privater Aufführungen. Und natürlich reagieren auch die Dichter auf diese neue Situation, dass sie nun nicht mehr ausschließlich für Hörer schreiben, sondern auch, sogar vorzugsweise, für Leser. ${ }^{9}$

Dieser zuletzt genannte Aspekt ist mir besonders wichtig: Durch die Verschriftlichung, die Sammlung und Kanonisierung literarischer Texte verändern sich nicht nur die Rezeptionsformen von Dichtung, sondern auch ihre Produktionsbedingungen. An drei Stichpunkten möchte ich illustrieren, wie sich hier aus einer Aufführungskultur tatsächlich das entwickelt, was auf vielen Wegen und Umwegen dann zu unserer modernen Vorstellung von Literatur führt:

1. Durch die Archivierung und Kanonisierung von Texten entstand eine klassische Tradition, die Lesern unmittelbar zur Verfügung stand und vor deren Hintergrund sich jeder neue Autor positionieren musste. Das erlegte Schriftstellern einerseits Druck auf: Wir hören nun Klagen darüber, es sei schwer, noch etwas Neues zu schreiben; alles Wichtige sei schon gesagt. ${ }^{10}$ Es gab ihnen andererseits Freiheit zum Experimentie-

6 Zu ihr vgl. Erskine (1995).

7 Johnstone (2014) geht in seiner Skepsis zu weit, wenn er die Existenz der Bibliothek von Alexandria im 3. Jh. bezweifelt; seiner Ansicht nach wurde die Bibliothek erst im 2. Jh. gegründet.

8 Downey (1962) 55.

9 Dazu vgl. vor allem Bing (2008).

10 So schon Ende des 5. Jh. bei Choirilos von Samos, frg. 317 Supplementum Hellenisticum. 
ren: Wo etwa dichterische Gattungen zuvor durch Aufführungskontexte dominiert waren, werden sie jetzt durch formale und inhaltliche Kriterien bestimmt; damit werden Autoren frei darin, ihre Genres zu wählen und mit ihnen spielerisch umzugehen. Solches Experimentieren mit Gattungskonventionen findet sich zahlreich in der gesamten hellenistischen Literatur.

2. Damit werden Dichter auch zu Lesern, und oft genug zu professionellen Lesern: Eine ganze Reihe von hellenistischen Autoren war an Bibliotheken als Philologen tätig, so besonders in Alexandria. Wenn solche gelehrte Autoren schreiben, dann tun sie das von Büchern umgeben, mit ständigem Blick auf den Kanon, den sie interpretieren, zitieren und kommentieren, auf den sie anspielen. Und sie erwarten auch von ihrem Publikum eine ähnliche Belesenheit: Ihre Texte wollen entschlüsselt, nicht einfach konsumiert werden. So ist es kein Zufall, dass auch ihre Bücher bereits wenige Generationen nach ihrer Produktion kommentiert werden wie die Klassiker: ${ }^{11}$ Damit ist die Integration in den Kanon vollendet; anspruchsvolle Literatur entsteht aus der Begegnung mit der Tradition und schreibt sich in diese Tradition ein in Erwartung zukünftiger Leser und Schriftsteller, die diese Traditionslinien fortführen werden.

3. Diese Einschreibung in die Tradition ist eng verbunden mit dem veränderten Publikum der hellenistischen Dichter: Ihre Dichtung richtet sich nicht mehr ausschließlich oder auch nur in erster Linie an eine ihnen zumindest prinzipiell bekannte Gemeinschaft von Mitbürgern oder eine begrenzte Festgesellschaft, sondern sie schreiben (auch) für ein anonymes, durch die ganze hellenistische Welt verstreutes Lesepublikum, das eben durch die Entschlüsselung und Interpretation dieser anspruchsvollen Texte erst zu einer virtuellen Gemeinschaft, einer „imagined community“ im Sinne von Anderson ${ }^{12}$ wird. Wenn solche Schriftsteller an die Rezipienten ihrer Texte denken, dann schauen sie weit über ihren geografischen und historischen Rahmen hinaus: Als Leser, Philologen und Bibliothekare wissen sie aus eigener Anschauung, dass Klassiker auch nach Jahrhunderten noch lebendig sind, daher erwarten sie für ihre eigenen Schriften ebenfalls ein solches Fortleben.

Eben in diese Phase der Verschriftlichung und Kanonisierung, der Etablierung einer Lesekultur und der Institutio-

11 S. dazu Montanari (1995).

12 Anderson (1991). nalisierung von Bibliotheken fällt die Kulturbegegnung Roms mit Griechenland..$^{13}$ Alexanders Eroberungen hatten dafür gesorgt, dass sich der Osten weithin hellenisierte; nach Italien war er nicht gekommen - was den militärisch unterlegenen Griechen später immerhin die Befriedigung gab, sich vorzustellen, Alexander hätte Rom leicht erobern können, wenn er es nur gewollt hätte. ${ }^{14}$ Kontakte der Römer mit griechischer Kultur erfolgten in mehreren großen Wellen: In der römischen Frühzeit (7.-4. Jh. v.Chr.) waren sie zunächst vor allem durch die Etrusker vermittelt, die ihrerseits zahlreiche griechische Kultureinflüsse empfangen hatten. Die römische Expansion seit dem 3. Jh. v. Chr. brachte sie in militärischen und friedlichen Kontakt mit den griechischen Städten Süditaliens und Siziliens. Seit dem 2. Jh. v. Chr. griff Rom als aufstrebende Macht im Mittelmeerraum zunehmend auch in die Auseinandersetzungen in Griechenland selbst und Kleinasien ein; in diesen Kontext gehört die zu Beginn des Beitrags angeführte Schlacht zwischen dem makedonischen König Perseus und Aemilius Paullus.

Selbstverständlich war die Literatur nicht das einzige Gebiet, auf dem die Römer eine im Vergleich zu ihrer eigenen weit fortgeschrittene griechische Zivilisation kennenlernten, rasch übernahmen und adaptierten. Wenn etwa Vergil (70-19 v.Chr.) in einer berühmten Passage seiner Aeneis (6, 847-853) der Kriegs- und Staatskunst Roms die Künste ,anderer“ (alii, womit klar die Griechen gemeint sind) gegenüberstellt, so nennt er Bildhauerei, Rhetorik und Astronomie. Und überraschend lange bleibt das Verhältnis der Römer gegenüber dieser griechischen Zivilisation ambivalent: ${ }^{15}$ Auf der einen Seite steht etwa die bekannte Formulierung von Vergils Zeitgenossen Horaz (Epistel 2, 1, 156f.), das eroberte Griechenland habe seinerseits den wilden Sieger erobert und in das bäurische Latium die Künste eingeführt; auf der anderen zieht sich eine kontinuierliche Linie von dem älteren Cato (234-149 v. Chr.), der in einem Brief seinen Sohn warnt, griechische Kultur werde „alles korrumpieren“ (zitiert bei Plinius, $\mathrm{Na}$ turalis Historia 29, 14), bis zu dem Satiriker Juvenal (frühes 2. Jh. n. Chr.), der das vorlaute und nichtsnutzige „Griechlein“ (Graeculus, Satire 3, 78) karikiert, das alles zu wissen vorgibt.

In mehreren Wellen fand auch die Begegnung mit der griechischen Literatur statt. Als ein Epochendatum der

$13 \mathrm{Zu}$ dieser folgenschweren Begegnung vgl. etwa Vogt-Spira et al. (1999).

14 So sagt Alexander in einem von Lukians Totengesprächen (25, 6; geschrieben im 2. Jh. n. Chr.), er habe sich nach Osten gewandt, weil die Völker im Westen ihm zu unbedeutend und schwach erschienen. 15 Vgl. dazu etwa Hinds und Schmitz (2007). 
lateinischen Literatur gilt das Jahr 240 v. Chr., als Livius Andronicus, ein Kriegsgefangener aus der süditalienischen Stadt Tarent, zum ersten Mal Dramen in lateinischer Sprache auf die Bühne bringt; er ist es auch, der die lateinische Epik durch seine Übersetzung der homerischen Odyssee begründet. Schon hier ist deutlich, dass diese Adaptationen auf schriftlichen Zeugnissen beruhen: Livius schafft einen geschriebenen Text aus einer ebenfalls geschriebenen Vorlage. Solche übersetzerische Tätigkeiten bleiben noch lange Zeit eine beliebte Art der Auseinandersetzung mit der griechischen Literatur, wenn etwa der komische Dichter Plautus (ca. 250-184 v. Chr.) in Prologen darauf hinweist, er habe griechische Komödien in das „barbarische“ Latein übersetzt (Maccus uortit barbare, Asinaria 11, vgl. Trinummus 19), ${ }^{16}$ wenn Catull Gedichte von Sappho und Kallimachos überträgt oder Cicero Dialoge Platons oder das astronomische Lehrgedicht des Arat. ${ }^{17}$

Doch schon bald setzt eine deutlich komplexere Art der Aneignung von und Auseinandersetzung mit griechischer Literatur ein: Lateinische Autoren schreiben als „gelehrte Dichter“ (poetae docti) anspruchsvolle Texte, deren Verständnis auch bei ihrem Publikum extensive und detaillierte Kenntnisse der (griechischen) Vorbilder voraussetzt; sie übertragen also das in der hellenistischen Literatur so prägende spannungsvolle Verhältnis zwischen Innovation und Tradition auf ihre eigene dichterische Produktion. ${ }^{18}$ Insbesondere in der augusteischen Periode entsteht auf diese Weise eine voraussetzungsreiche und raffinierte Literatur, deren Funktionsweise auf der Bereitschaft und Fähigkeit des Publikums beruht, Anspielungen auf griechische Texte zu entschlüsseln und diese Art indirekter literarischer Kommunikation zu schätzen. Wenige Generationen später rücken dann die Texte dieser augusteischen Dichtung ihrerseits in die Position klassischer Referenzen, auf die sich spätere Autoren beziehen; es entsteht neben der griechischen auch eine genuin lateinische Tradition.

Auf diese Weise entsteht etwa seit dem zweiten vorchristlichen Jahrhundert in Rom eine genuin zweisprachige Kultur: ${ }^{19}$ Griechische Gelehrte, Grammatiker, Rhetoriklehrer, Philosophen und Wissenschaftler sind in Rom tätig

16 Die Frage, wieviel von den Komödien des Plautus tatsächlich Übersetzung ist und wieviel auf italische Traditionen zurückgeht, wird in der latinistischen Forschung seit langem intensiv diskutiert; eine gute Annäherung bietet Fontaine (2014).

17 Einen knappen Überblick über diese Übersetzungstätigkeiten bieten Lewis (1986) und Feeney (2016) 45-91.

18 Aus der immensen Forschungsliteratur der letzten Jahrzehnte seien lediglich einige wenige Beispiele genannt: Hinds (1998), Thomas (1999), Schmitz (2015).

19 Dazu etwa Swain (2004). und finden dort ein eifriges (und zahlungskräftiges) Publikum; Angehörige der römischen Eliten beherrschen Griechisch wie eine zweite Muttersprache, haben Unterricht sowohl bei lateinischen wie bei griechischen Lehrern, und verbringen oftmals Jahre des Auslandsstudiums in Athen oder Kleinasien; ${ }^{20}$ manche von ihnen verfassen sowohl griechische als auch lateinische Werke. Nicht immer hat die spätere Überlieferung diese Zweisprachigkeit gebührend gewürdigt, wie an einem kleinen Beispiel gezeigt sei: Als Caesar am 10. Januar 49 v. Chr. den Rubicon an der Spitze eines Heeres überschreitet und sich damit außerhalb der römischen Legalität stellt, unterstreicht er die Tragweite dieses entscheidenden Schrittes, der den römischen Bürgerkrieg einleitet, durch ein markantes Zitat. In das kollektive Gedächtnis eingegangen ist es in seiner lateinischen, bei Sueton (Iulius 32) überlieferten Form iacta alea est. Doch Caesar tat, wie Plutarch (Pompeius 60, 4:

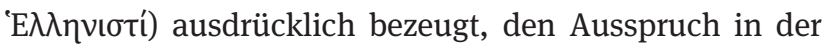
griechischen Form óvveppi $\varphi \theta \omega$ kúßos: Es handelt sich um ein Zitat aus dem griechischen Komiker Menander (3. Jh. v. Chr.; frg. 59, 4 Koerte), zu dem Plutarch in seiner Biografie Caesars $(32,8)$ und Appian in seiner Geschichte der Römischen Bürgerkriege $(2,5,35)$ ausführen, es sei bei allen sprichwörtlich, die sich in große Risiken stürzten. Caesars Ausspruch war nicht nur für spätere Zeiten traditionsbildend, sondern bezog sich bereits seinerseits auf eine lange Tradition, die den Zeitzeugen völlig geläufig war.

Bibliotheken spielen bei dieser Etablierung einer zweisprachigen literarischen Kultur eine zentrale Rolle. Dass die von Aemilius Paullus nach Rom gebrachten Bücher des Perseus die erste große Bibliothek in Rom darstellten, wird zwar bei Plutarch nicht ausdrücklich gesagt, scheint aber eine plausible Vermutung, auch wenn das einzige Zeugnis, das diese Information gibt, der spätantike Enzyklopädist Isidor von Sevilla (Etymologiae 6, 5, 1), nicht gerade als besonders zuverlässig gilt. ${ }^{21}$ Die Söhne des Aemilius Paullus genossen offenbar Unterricht bei griechischen Lehrern und waren deshalb „Literaturliebhaber“ ( thek nicht mehr profitieren konnten, war in Rom jetzt eine große Sammlung griechischer Texte verfügbar. Und es sollte nicht die einzige bleiben: In erstaunlicher Geschwindigkeit wurden Bibliotheken zu einem selbstverständlichen Bestandteil des römischen Kulturlebens.

Als ein Beispiel dafür, wie Bibliotheksbenutzung Teil des Alltags eines gebildeten Römers war, sei eine Passage aus Ciceros 45 v. Chr. entstandenem Werk Über das

20 Vgl. Howley (2014). 21 Vgl. Affleck (2013) $124 \mathrm{ff}$. 
höchste Gut und das größte Übel angeführt. Cicero vermittelt hier einem römischen Publikum eine Fachdiskussion der griechischen Philosophie: Was ist das Ziel des menschlichen Lebens? Und welche Rolle spielt Unglück für die Erreichung dieses Ziels? Zu Beginn des dritten Buchs schildert Cicero, wie er mit Marcus Cato in eine philosophische Diskussion geriet $(3,7):^{22}$

\begin{abstract}
„nam in Tusculano cum essem uellemque e bibliotheca pueri Luculli quibusdam libris uti, ueni in eius uillam, ut eos ipse, ut solebam, depromerem. quo cum uenissem, M. Catonem, quem ibi esse nescieram, uidi in bibliotheca sedentem multis circumfusum Stoicorum libris. erat enim, ut scis, in eo auiditas legendi, nec satiari poterat, quippe qui ne reprehensionem quidem uulgi inanem reformidans in ipsa curia soleret legere saepe, dum senatus cogeretur, nihil operae rei publicae detrahens. quo magis tum in summo otio maximaque copia quasi helluari libris, si hoc uerbo in tam clara re utendum est, uidebatur."

„Als ich auf meinem Landgut in Tusculum war und einige Bücher aus der Bibliothek des jungen Lucullus benutzen wollte, ging ich in seine Villa, um sie selbst herauszuholen, wie ich es häufig tat. Als ich dorthin kam, fand ich Marcus Cato, von dessen Anwesenheit ich nichts geahnt hatte, in der Bibliothek sitzen, umgeben von vielen Schriften der stoischen Philosophen. Er war, wie du weißt, dermaßen begierig auf das Lesen und unersättlich darin, dass er sogar, ohne die unbegründete Kritik der Menge zu fürchten, in der Curie selbst zu lesen pflegte, während der Senat sich versammelte, ohne deswegen etwa seinen öffentlichen Verpflichtungen weniger zu genügen. Umso mehr schien er in dieser Zeit der Muße und in einer solchen Fülle geradezu zu schwelgen in den Büchern, wenn man denn bei einer so ausgezeichneten Sache diesen Ausdruck verwenden darf.“
\end{abstract}

Besonders in den Jahren seiner erzwungenen politischen Muße nach 47 v. Chr., als Caesar die Alleinherrschaft in Rom innehatte, schrieb Cicero in rascher Folge eine Reihe philosophischer Werke, in denen er dem römischen $\mathrm{Pu}$ blikum die Grundzüge der griechischen Philosophie zu vermitteln suchte; ${ }^{23}$ dazu benötigte er Zugang zu spezialisierter Literatur. Die große Bibliothek des Lucullus hatte umfangreiche Bestände solcher philosophischer Literatur; wiederum Isidor von Sevilla (Etymologiae 6, 5) überliefert, der Vater des jungen Mannes habe sie als Kriegsbeute von seinen Feldzügen im Osten heimgebracht, ähnlich wie etwa ein Jahrhundert zuvor Aemilius Paullus die Bibliothek des Perseus. Cicero und Cato benutzen diese Bibliothek in Abwesenheit des Hausherren offenbar wie ihre eigene; Plutarch (Lucullus 42, 1-2) berichtet davon, der ältere Lucullus habe seine Bibliothek zu einem Ort der Begegnung und des Gesprächs gemacht, in dem besonders griechische Intellektuelle sich zwanglos zusammenfinden konnten und der Hausherr sich oftmals in die Diskussionen einschaltete.

Waren also solche privaten, teils auch umfangreiche Bibliotheken in der römischen Gesellschaft bereits seit der Republikzeit verbreitet, gewinnen sie seit dem Beginn der Kaiserzeit noch einen ganz anderen Stellenwert: Die römischen Kaiser gründen seit dem 1. Jh. n. Chr. eine Reihe von öffentlichen Bibliotheken in Rom, die in der Regel sowohl griechische als auch lateinische Bestände umfassten. Julius Caesar konnte seinen Plan, eine große zweisprachige Bibliothek einzurichten (Sueton, Iulius 44), nicht mehr durchführen, doch sein Nachfolger und Adoptivsohn $\mathrm{Au}$ gustus gründet gleich zwei Bibliotheken, eine im Bezirk des Apollon auf dem Palatin, eine weitere in der Porticus Octaviae. ${ }^{24}$ Eine weitere bedeutende Bibliothek in Rom, die Ulpiana, gründet Traian zu Beginn des 2. Jh. n.Chr. In dieser Zeit verfügt Rom über eine ganze Reihe privater und öffentlicher Sammlungen, die große Bestände haben, und selbst seltene Werke sind in mehreren Exemplaren in der Stadt vorhanden. Als Beispiel seit etwa eine Passage aus einem Brief des späteren Kaisers Marc Aurel an seinen Erzieher, den Gelehrten Fronto, zitiert. Marc Aurel hat sich auf das Land zurückgezogen und dorthin zur Lektüre und als Vorbild für eigene Erzeugnisse zwei Reden des älteren Cato mitgenommen; Fronto und er sind an solchen Zeugnissen für die frühe Latinität besonders interessiert. Er malt sich aus, wie Fronto nach Exemplaren dieser Reden sucht (Fronto, Epistulae ad M. Caesarem et invicem 4, 5, 2):
„Legi Catonis orationem de Bonis Pulchrae et aliam, qua tribuno diem dixit. "io," inquis puero tuo, "uade quantum potes, de Apollinis bibliotheca has mihi orationes adporta." frustra: nam II isti libri me secuti sunt. igitur Tiberianus bibliothecarius tibi subigitandus est; aliquid in eam rem insumendum, quod mihi ille, ut ad urbem uenero, aequa diuisione inpertiat. sed ego orationibus his perlectis paululum misere scripsi, quod aut Lymphis aut Volcano

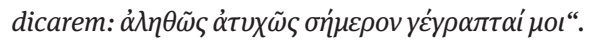

„Ich habe Catos Rede Über die Güter der Pulchra gelesen und eine andere, in der er einen Tribunen anklagte. ,Los‘, sagst du zu deinem Diener, ,geh, so schnell du kannst, und hole mir diese Reden aus der Apollonbibliothek!' Du schickst ihn vergeblich, denn diese beiden Bücher sind mir gefolgt. Deshalb musst du den Bibliothekar der Tiberiana herumkriegen; dafür musst du ein bisschen was springen lassen, das er, sobald ich wieder nach Rom zurückkehre, gerecht teilen soll! Nach der Lektüre dieser Reden habe ich mühselig ein bisschen etwas geschrieben, das ich den Gottheiten des Wassers oder des Feuers weihen werde: Mein Schreiben heute war wirklich ohne jeden Erfolg“.
22 Vgl. zu dieser Passage Tutrone (2013) $157 \mathrm{ff} .$, zur Bibliothek des Lucullus Dix (2000).

23 Vgl. dazu etwa Leonhardt (1999).
24 Vgl. Blanck (1992) 162f. Zu Recht weist Marshall (1976) $262 \mathrm{ff}$. darauf hin, dass dieses kaiserliche Patronat für Bibliotheken zugleich auch eine Kontrolle über Literatur bedeutete. 
Die Exemplare aus der Palatinsbibliothek hat Marcus mit sich genommen; wenn Fronto, als gewissenhafter Erzieher, die Werke ebenfalls lesen will, um mit seinem Schüler darüber zu fachsimpeln, muss er in die (der Öffentlichkeit eigentlich nicht zugängliche) Tiberiana im Kaiserpalast gehen, und Marcus stellt sich scherzhaft vor, wie Fronto den Bibliothekar dort bestechen muss, um eingelassen $\mathrm{zu}$ werden, und er nach seiner Rückkehr das Geld mit ihm teilen wird. Lektüre bedeutet für Marcus, wie rund zwei Jahrhunderte vorher für Cicero, nicht lediglich Rezeption, sondern auch Vorbereitung zu eigenem Schreiben: Angeregt durch Catos Reden hat auch er etwas geschrieben, das allerdings nur dazu taugt, mit dem Schwamm ausgewischt oder verbrannt zu werden.

In bescheidenerem Umfang gab es solche Bibliotheken nicht nur in der Hauptstadt, sondern auch in der Provinz. Ein besonders beeindruckender Fall ist die umfangreiche philosophische Bibliothek in Herculaneum, die der Vesuvausbruch 79 n. Chr. über die Jahrhunderte konserviert hat und die seit den Anfängen der Ausgrabungen im 18. Jh. bis heute noch nicht alle ihre Geheimnisse preisgegeben hat. ${ }^{25}$ Bemerkenswert ist auch die von Plinius d.J. nur wenig später in seinem Heimatstädtchen Comum gegründete Bibliothek, über die wir nicht nur aus einem seiner Kunstbriefe informiert sind (1, 8: Plinius bittet einen Freund, die bei der Einweihung der Bibliothek gehaltene Rede für die Publikation durchzusehen), sondern auch aus seiner Grabinschrift, die stolz vermerkt, dass er für ihre Gründung eine Million Sesterzen aufgewendet hat und für ihren Unterhalt 100000 jährlich hinterlässt. ${ }^{26}$ Mochte auch die Hauptstadt für einen Literaten oder Wissenschaftler die besten Arbeitsbedingungen bieten, selbst auf dem Land war man von Bibliotheken nicht völlig abgeschnitten. ${ }^{27}$

Besonders relevant scheint mir, dass durch solche großen Sammlungen seit dem 2. Jh. v. Chr. in Rom die griechische Literatur und Philosophie auf einen Schlag in ihrer Gesamtheit als gewaltiges kulturelles Archiv verfügbar war. Lateinische Autoren hatten in diesen Bibliotheken nicht lediglich Zugang zu mehr oder minder zufällig zusammengewürfelten Beständen, sondern zum Panorama einer Tradition, die sie als einheitlich empfinden und aus der sie jeweils relevante Bestandteile für ihre eigenen Produktionen als Vorbilder, Intertexte und Ausgangspunkte auswählen konnten. Die vorhandenen materiellen Bibliotheken lassen sich als Verkörperung dieser ideellen Bib-

25 Zur ersten Einführung s. Sider (2009).

26 CIL 5, 5262 = ILS 2927, am bequemsten zugänglich in SherwinWhite (1966) 732.

27 Vgl. Blanck (1992) $156 \mathrm{ff}$. liothek griechischer Literatur verstehen, die für römische Autoren und Leser Anstöße zu Nachahmung und Wetteifern (imitatio et aemulatio) gab. Diese ideelle Bibliothek wurde Stück für Stück in die lateinische Sprache und die römische Kultur übertragen: Römische Autoren hatten aus dieser Fülle literarischer Möglichkeiten die Wahl zwischen verschiedenen poetischen Genres, zwischen einer Reihe von Vorbildautoren und -texten. So erklären sich die zahlreichen Passagen, in denen ein Dichter beansprucht, als erster „parische Iamben“ (Horaz, epistula 1, 19, 23-24; d. h. Spottdichtung in der Art des Archilochos aus Paros) oder äolische Lyrik nach Italien gebracht zu haben (Horaz, carmen 3, 30, 13-14) oder der neue Alkaios, Kallimachos oder Mimnermos zu sein (Horaz, epistula 2, 2, 99-101): Dahinter steht die Vorstellung einer großen Tradition, einer imaginären Bibliothek, die man Stück für Stück adaptiert, sich zu eigen macht, als eigenes Erbe deklariert.

Seit der hellenistischen Zeit finden wir in griechischen Texten Passagen, in denen sich Autoren als Schreibende darstellen und auf die Materialität ihres Schaffensprozesses Bezug nehmen, etwa im Proöm der Aitien des Kallimachos (frg. 1, 21-22; 3. Jh. v. Chr.) oder im Froschmäusekrieg (1-3; wahrscheinlich 1. Jh. n.Chr.). ${ }^{28}$ So ausführliche Darstellungen des materiellen Gedichtbuchs wie in der lateinischen Literatur aber findet man auf der griechischen Seite kaum, wenn etwa Horaz am Ende des ersten Buchs seiner poetischen Episteln $(1,20)$ das Schicksal der Buchrolle bei Händlern und Lesern schildert oder Ovid in mehreren Elegien seiner Tristien $(1,1 ; 3,1)$ den Weg der Buchrollen aus dem Exil in Tomi in die Hauptstadt Rom imaginiert. ${ }^{29}$ Will man über den Grund dafür spekulieren, dass das Buch und seine äußere Form für lateinische Autoren eine solch prägende Rolle gespielt hat, so wird man wieder auf die griechischen Vorbilder verweisen: Das bestimmende Bild der literarischen Tradition war für einen römischen Dichter das Buch und die Bibliothek; ein Autor zu sein bedeutete, schriftliche Werke zu verfassen und sich ganz wörtlich in diese Tradition einzuschreiben.

Wenn wir zum Abschluss dieses gedrängten Überblicks die Bedeutung von (griechischen) Büchern und Bibliotheken für die Entstehung einer lateinischen Literatur zusammenfassend verstehen wollen, so können wir das vielleicht mit dem Mittel der kontrafaktischen Geschichte erreichen. Eine solche Geschichtsbetrachtung lädt dazu ein, uns einen alternativen Verlauf der Geschichte vorzustellen, wenn entscheidende Schlüsselereignisse anders als in der Realität verlaufen wären: Wie sähe Europa heute

28 Vgl. Bing (2008) 17-48.

29 Vgl. zu diesen Texten etwa Dupont (2009) 152-160. 
aus, wenn Franz-Ferdinand 1914 das Attentat von Sarajevo überlebt hätte? Oder wenn 480 v. Chr. nicht die Griechen, sondern die Perser in der Schlacht von Salamis gesiegt hätten?

Unsere Fragen für eine solche alternative Geschichte lauten: Wie sähe die Welt aus, wenn Alexander seine Eroberungen ein Jahrhundert früher gemacht hätte, vor der großen Phase der Verschriftlichung griechischer Dichtung? Auch dann wäre wahrscheinlich in den von ihm eroberten Gebieten griechische Kunst, Architektur, Städteplanung, Wissenschaft, vielleicht auch Philosophie attraktiv gewesen und hätte Menschen in ihren Bann gezogen. Aber eine globalisierte griechische Literatur hätte es wohl nicht gegeben, weil sich eine ganz auf Performance und Gesang aufgebaute Kultur nicht so gut verpflanzen lässt wie ein geschriebenes Buch; eine literarische Tradition, die Schriftstellern überall in der hellenisierten Welt Anregungen zur Auseinandersetzung und zum Weiterschreiben, dem Publikum einen Erwartungshorizont zur Einordnung neuer Werke geboten hätte, wäre in dieser Konstellation wohl nicht entstanden.

Einen ähnlichen Befund erreichen wir mit einer anderen alternativen Geschichtsversion: Wie sähe unsere Welt aus, wenn die Römer Griechenland ein Jahrhundert früher erobert hätten, wenn Aemilius Paullus seinen Söhnen Statuen und Silberpokale, aber eben keine Bibliothek hätte mitbringen können? Auch hier wäre meine tentative Antwort: Eine Gesangskultur hätte die Römer nicht so nachhaltig beeindruckt wie die plötzliche und massive Verfügbarkeit einer avancierten Literatur. Ohne Bibliotheken und Bücher hätten sie das Ideal des poeta doctus, des gelehrten Dichters, der für ein gebildetes Publikum schreibt, nicht übernehmen können. Literatur und Philosophie wären nicht als prestigebringende Tätigkeiten einer kulturellen Elite zutage getreten.

Gäbe es auch in diesen alternativen Welten heute, mehr als 2000 Jahre nach diesen Ereignissen, Literatur? Meine Antwort wäre: Es gäbe sie zumindest nicht in der Form, wie wir sie heute kennen. Von den literaturliebenden Söhnen des Aemilius Paullus, von Cicero in der Bibliothek des Lucullus bis zu unserem modernen Literaturbetrieb ist es ein großer Schritt; das Konzept des Autors und Dichters musste eine Reihe einschneidender historischer Entwicklungen durchmachen, von denen schlagwortartig lediglich die Begegnung der kulturellen Traditionen mit dem Christentum, der nahezu vollständige Verlust des antiken Bildungssystems in den Völkerwanderungen, der Umbau der antiken Kultur und ihrer Vermittlung im Mittelalter und die allmähliche Wiederentdeckung der antiken Literatur seit der Renaissance genannt seien. Dennoch scheint mir in all diesen Wandlungen und Um- formungen, Gefährdungen und Wiedergewinnungen als Modell anspruchsvoller Literatur die Begegnung der römischen Kultur mit der griechischen Tradition weiterzuleben.

Hier erreichen wir das Ziel der vorangegangenen Darstellung: Mir ging es darum zu zeigen, dass der Gang der Kulturgeschichte manchmal von ganz kontingenten Faktoren entscheidend geprägt wird. Alexander trägt die griechische Kultur just zu dem Zeitpunkt bis an die Grenzen der damals bekannten Welt, als sie durch die aufkommende Lesekultur leicht verpflanzbar geworden war. Die Römer begegnen dieser griechischen Literatur just zu dem Zeitpunkt, als sie in Bibliotheken als schriftliche Tradition verfügbar war, als sich die griechische Kultur durch die Eroberungen Alexanders globalisiert hatte und damit leichter adaptierbar geworden war, als das Modell des gelehrten, schriftlich wirkenden Autors entwickelt worden war. Beide Zufälle zusammen haben dafür gesorgt, dass Literatur so wurde, wie sie heute ist.

\section{Literaturverzeichnis}

Affleck, Michael (2013): Priests, patrons, and playwrights. Libraries in Rome before 168 BC. In: Ancient Libraries, ed. by Jason König, Katerina Oikonomopoulou und Greg Woolf. Cambridge: Cambridge University Press, 124-36.

Anderson, Benedict (1991): Imagined communities. Reflections on the origin and spread of nationalism. 2. Aufl. London: Verso.

Bing, Peter (2008): The well-read Muse. Present and past in Callimachus and the Hellenistic poets. 2. Aufl. Ann Arbor: Michigan Classical Press.

Blanck, Horst (1992): Das Buch in der Antike. München: Beck.

Dix, T. Keith (2000): The Library of Lucullus. In: Athenaeum, 88, 441-64.

Downey, Glanville (1962): Ancient Antioch. Princeton: Princeton University Press.

Dupont, Florence (2009): The corrupted boy and the crowned poet or, the material reality and the symbolic status of the literary book at Rome. In: Ancient literacies. The culture of reading in Greece and Rome, ed. by William A. Johnson and Holt N. Parker. Oxford: Oxford University Press, 143-63.

Erskine, Andrew (1995): Culture and power in Ptolemaic Egypt. The museum and library of Alexandria. In: Greece \& Rome, 42, 38-48.

Feeney, Denis (2016): Beyond Greek. The beginnings of Latin literature. Cambridge (Mass.): Harvard University Press.

Fontaine, Michael (2014): Between two paradigms: Plautus. In: The Oxford handbook of Greek and Roman comedy, ed. by Michael Fontaine and Adele C. Scafuro. Oxford: Oxford University Press, 516-37.

Herington, John (1985): Poetry into drama. Early tragedy and the Greek poetic tradition, Berkeley: University of California Press.

Hinds, Stephen (1998): Allusion and inter text. Dynamics of appropriation in Roman poetry. Cambridge: Cambridge University Press. 
Hinds, Stephen; Schmitz, Thomas A. (2007): Constructing identities in the Roman Empire: three studies. In: Millennium, 4, 1-12.

Howley, Joseph A. (2014): “Heus tu rhetorisce”: Gellius, Cicero, Plutarch, and Roman study abroad. In: Roman rule in Greek and Latin writing. Double vision, ed. by Jesper M. Madsen and Roger Rees. Leiden: Brill (Impact of Empire: 18), 163-92.

Immerwahr, Henry R. (1964): Book rolls on Attic vases. In: Classical, Mediaeval, and Renaissance Studies in Honor of Berthold Louis Ullman, ed. by Charles Henderson. Rom: Edizioni di storia e letteratura, 17-48.

Immerwahr, Henry R. (1973): More book rolls on Attic vases. In: Antike Kunst, 16, 143-47.

Johnstone, Steve (2014): A new history of libraries and books in the Hellenistic world. In: Classical Antiquity, 33 (2), 347-93.

Leonhardt, Jürgen (1999): Ciceros Kritik der Philosophenschulen. München: Beck (Zetemata: 103).

Lewis, Anne-Marie (1986): Latin translations of Greek literature: the testimony of Latin authors. In: L'Antiquité Classique, 55, 163-74.

Marshall, Anthony J. (1976): Library resources and creative writing at Rome. In: Phoenix, 30, 252-64.

Montanari, Franco (1995): Filologi alessandrini e poeti alessandrini. La filologia sui contemporanei. In: Aevum antiquum, 8, 47-63.

Pébarthe, Christophe (2006): Cité, démocratie et écriture. Histoire de l'alphabétisation d'Athènes à l'époque classique. Paris: de Boccard.

Powell, Barry B. (2009): Writing: theory and history of the technology of civilization, Chichester: Wiley-Blackwell.

Schmitz, Thomas A. (2015): Allusion, Intertext, Zitat. Die Vergilforschung und neuere Tendenzen der Literaturwissenschaft. In: Gymnasium, 122, 525-57.

Sherwin-White, A. N. (1966): The letters of Pliny. A historical and social commentary. Oxford: Oxford University Press.
Sider, David (2009): The special case of Herculaneum. In: The Oxford Handbook of Papyrology, ed. by Roger S. Bagnall. Oxford: Oxford University Press, 303-19.

Swain, Simon (2004): Bilingualism and biculturalism in Antonine Rome. Apuleius, Fronto, and Gellius. In: The Worlds of Aulus Gellius, ed. by Leofranc Holford-Strevens and Amiel Vardi. Oxford: Oxford University Press, 3-40.

Thomas, Richard F. (1999): Reading Virgil and his texts: Studies in intertextuality. Ann Arbor: University of Michigan Press.

Tutrone, Fabio (2013): Libraries and intellectual debate in the late Republic. The case of the Aristotelian corpus. In: Ancient Libraries, ed. by Jason König, Katerina Oikonomopoulou and Greg Woolf. Cambridge: Cambridge University Press, 152-66.

Vogt-Spira, Gregor; Rommel, Bettina; Musäus, Immanuel (Hrsg.) (1999): Rezeption und Identität. Die kulturelle Auseinandersetzung Roms mit Griechenland als europäisches Paradeigma. Stuttgart: Steiner.

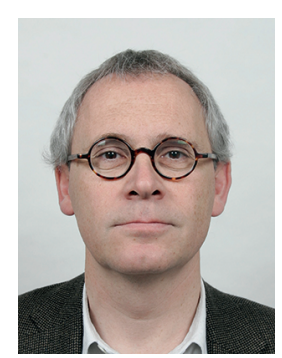

Prof. Dr. Thomas A. Schmitz

Rheinische Friedrich-Wilhelms-Universität Bonn

Philosophische Fakultät

Klassische und Romanische Philologie Am Hof 1

D-53113 Bonn

thomas.schmitz@uni-bonn.de 